\title{
Demain, le Photovoltaïque: Les révolutions anticipées sur les filières et les concepts
}

Jean-François Guillemoles (jean-francois.guillemoles@edf.fr) Institut de Recherche et Développement sur l'Énergie Photovoltaïque IRDEP, UMR 7174 CNRS-EDF-ENSCP, 11 Quai Watier, 78401 Chatou cedex

En principe, la conversion photovoltaïque de l'énergie solaire pourrait être beaucoup plus efficace qu'elle ne l'est actuellement avec de simples jonctions $p-n$. On en explique et discute ici les raisons.

Diverses stratégies d'approche de la limite de conversion photovoltaïque (87\%) sont ensuite présentées, en commençant par les dispositifs à multijonctions qui sont déjà utilisés dans les applications spatiales. D'autres filières, plus prospectives, sont les transformateurs optiques par addition ou division de photons,

les semi-conducteurs à niveaux intermédiaires, l'ionisation par impact et les cellules solaires à porteurs chauds.
Limitations et perspectives de la conversion photovoltaïque

Comme tout système de conversion d'énergie, un générateur photovoltaïque a ses performances limitées par les lois de la thermodynamique. Dans une première approche, on peut considérer le soleil comme une source chaude à $T_{c}=6000 \mathrm{~K}$ (sa température de surface), la source froide étant aux environs de $\mathrm{T}_{\mathrm{f}}=300 \mathrm{~K}$. Le rendement de Carnot associé à la conversion de l'énergie solaire peut donc en principe approcher $1-\mathrm{T}_{\mathrm{f}} / \mathrm{T}_{\mathrm{c}}=95 \%$ ! Or, les dispositifs photovoltaïques actuels, des photodiodes basées sur des matériaux semi-conducteurs, en sont loin avec des rendements de l'ordre de $20 \%$. La difficulté est de vouloir convertir de façon optimale un ensemble de photons dont l'énergie va de l'infrarouge au proche ultraviolet, avec un seul matériau actif dont les propriétés optiques ne sont bien adaptées qu'à une énergie de photon donnée (l'absorption n'étant importante qu'au-delà d'un seuil, $\mathrm{E}_{\mathrm{g}}$, dépendant du matériau choisi).

Pour comprendre pourquoi, il faut revenir aux principes de la conversion photovoltaïque [1], telle que les dispositifs actuellement réalisés la mettent en œuvre. Cette conversion se fait en trois étapes (fig. 1) : (i) absorption de photons d'énergie $h v>E_{g}$, qui crée des populations d'électrons et de trous hors équilibre ; (ii) chaque type de porteurs atteint très vite un quasi-équilibre défini par un quasi-niveau de Fermi (i.e. un potentiel électrochimique) $E_{f n}$ pour les électrons et $\mathrm{E}_{\mathrm{fp}}$ pour les trous, dont la différence, $\mathrm{E}_{\mathrm{fn}}-\mathrm{E}_{\mathrm{fp}}=\mathrm{qV}$, est l'énergie libre récupérable par photon absorbé (V est la phototension) ; (iii) les porteurs, collectés aux contacts avant d'avoir pu se recombiner, participent au photocourant. Au final, le dispositif photovoltaïque fonctionne d'une manière assez semblable à une batterie électrochimique un peu particulière : son anode et sa cathode sont constituées par des niveaux d'énergie différents du même matériau!

Or, dans ce type de dispositif, on doit avoir $h v>E_{g} d u$ fait des propriétés d'absorption, mais aussi $E_{\mathrm{g}}>\mathrm{E}_{\mathrm{fn}}-\mathrm{E}_{\mathrm{fp}}{ }^{(1)}$. La fraction d'énergie qui peut être efficacement convertie, égale à $\mathrm{qV} / \mathrm{h} v$, est de ce fait relativement réduite, car on n'absorbe pas une partie du spectre et parce que l'énergie libre obtenue de chaque photon est indépendante de l'énergie du photon absorbé ; le reste est cédé au réseau atomique en moins de quelques picosecondes, sous forme de chaleur lors de la thermalisation des porteurs. Le rendement de
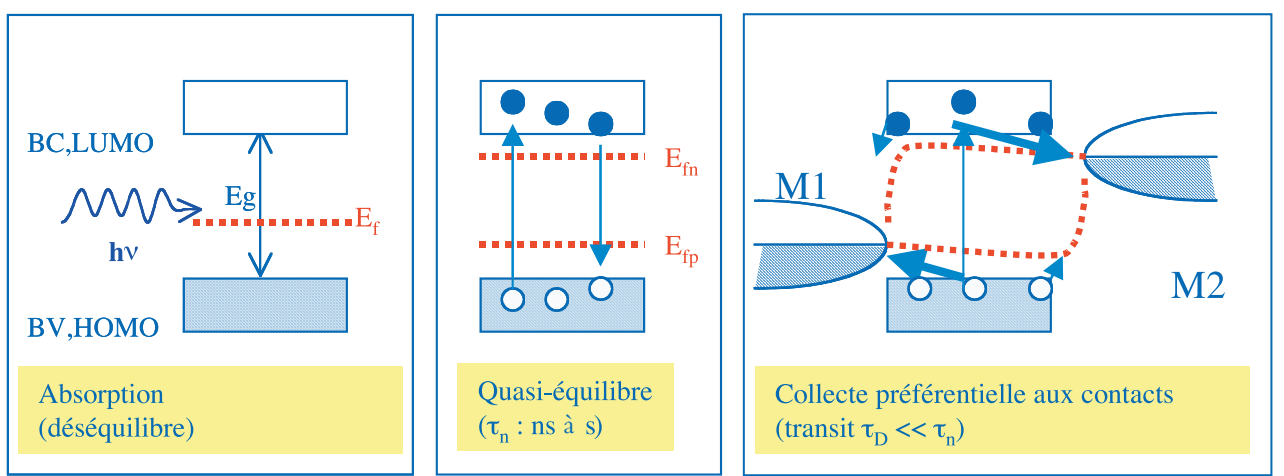

Figure 1 : Principe de la conversion photovoltaïque, décomposé en trois étapes principales. (i) Absorption d'un photon et promotion d'un électron sur un niveau vide supérieur. (ii) Établissement de deux populations d'électrons et de trous en quasi-équilibre interne (via des processus rapides de relaxation, à l'échelle de la ps), mais hors équilibre l'une par rapport à l'autre (les processus interbandes de retour à l'équilibre ont un temps caractéristique $\tau_{n}$, de l'ordre de la ns). (iii) Collecte préférentielle d'un type de porteur de charge à chaque contact après un temps de transit $\tau_{D} . M 1$ et $M 2$ sont les deux contacts, ici de type métallique, servant à la collecte, respectivement des trous et des électrons. La nature différente des deux contacts est à l'origine de la rupture de symétrie qui permet l'apparition d'une phototension. 


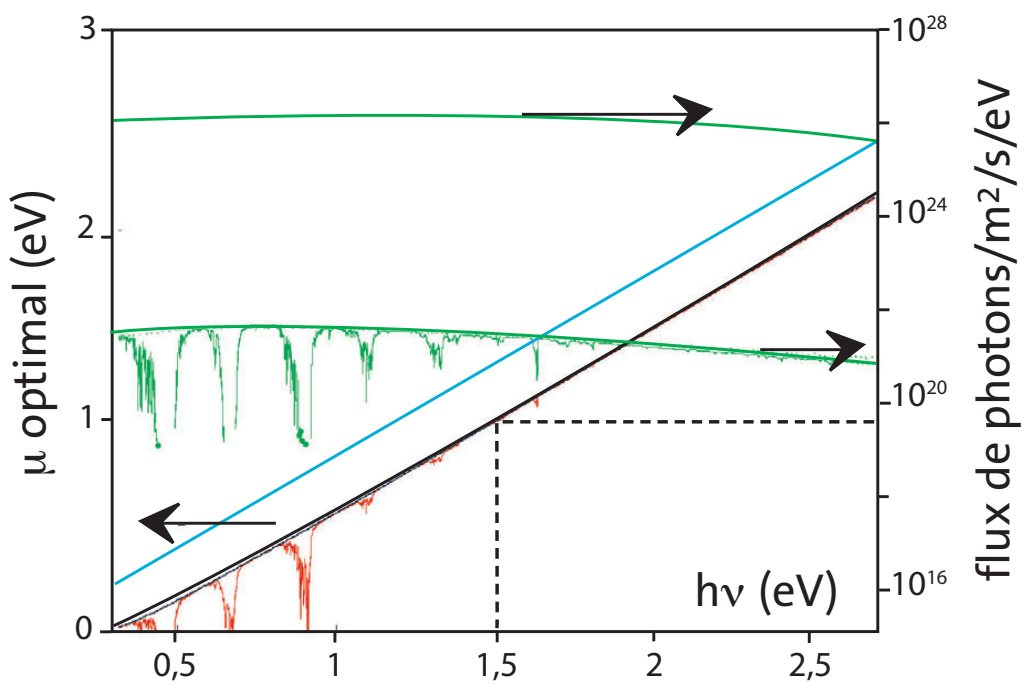

Figure 2 : Énergie libre optimale récupérable par photon absorbé, au maximum de rendement de conversion (ordonnée, axe de gauche), en fonction de l'énergie du photon (abscisse) et du nombre de photons absorbés. La courbe bleue correspond à un spectre de corps noir à $6000 \mathrm{~K}$, la courbe rouge à l'intensité solaire sur terre, et la courbe noire à celle du flux solaire avant son entrée dans l'atmosphère.

Courbes vertes: le flux de photons sur une échelle log (ordonnée, axe de droite) est donné pour les mêmes spectres.

La courbe en tirets noirs donne l'énergie libre récupérée par un photon dans une cellule solaire de seuil $E_{g}=1,5 \mathrm{eV}$, en fonction de l'énergie absorbée. Pour $h v>E_{g}$, elle est indépendante de l'énergie du photon absorbé.

(1) Lorsque $\mathrm{E}_{\mathrm{fn}}-\mathrm{E}_{\mathrm{fp}}$ devient proche de $\mathrm{E}_{\mathrm{g}}$, on atteint l'inversion de population entre électrons et trous, et l'émission stimulée de radiation vient s'opposer à ce que $\mathrm{qV}$ augmente encore.

(2) En général, $E_{f}=E_{f}{ }^{\circ}+k T \cdot \log [n]$, où $n$ est la concentration d'électrons du groupe de niveaux d'énergie considéré et $\mathrm{E}_{\mathrm{f}}{ }^{\circ}$ l'énergie libre de l'etat standard. C'est une expression similaire à celle du potentiel chimique d'un gaz parfait.

(3) Sinon, on pourrait transporter réversiblement de la chaleur d'un corps froid vers un corps chaud, et nous aurions déjà parmi nous des machines à mouvement perpétuel.

\section{Les filières à très hauts rendements}

On peut regrouper les stratégies d'augmentation des rendements en trois directions principales, toutes ayant des rendements de conversion ultimes voisins de $85 \%$.

1 - Dispositifs "photoniques » : si toute l'énergie solaire était concentrée dans une bande spectrale étroite, les dispositifs actuels seraient déjà capables d'en convertir plus de $50 \%$. On peut donc essayer d'adapter le spectre incident à une ou plusieurs photodiodes. Les exigences nouvelles portent donc ici sur les propriétés optiques des matériaux.

2 - Les matériaux à mécanismes d'absorption optimisés : on peut penser à des matériaux contenant des niveaux électroniques intermédiaires servant d' "échelle à électrons " ou à des matériaux permettant la génération de plusieurs paires électron-trou dès que le photon incident possède une énergie suffisante. Les exigences portent cette fois sur la structure électronique des matériaux.

3 - Les machines thermiques dans lesquelles l'absorption de lumière conduit bien à la production de chaleur (énergie cinétique électronique), mais où cette chaleur est convertie en puissance électrique. Dans ce dernier cas, il va falloir s'intéresser de près aux propriétés thermiques et " phononiques » des solides envisagés.

\section{I- Les dispositifs photoniques}

La difficulté vient de la mise en œuvre d'un travail en amont sur les photons qui sont soit triés en longueur d'onde pour être envoyés sur des jonctions adaptées à la partie du spectre correspondante (multijonctions), soit décalés en énergie par transfert d'une partie du spectre à une autre (tout en conservant l'énergie lumineuse totale) pour conduire à une répartition plus " ramassée ", avant d'être récupérés par une diode adaptée à ce nouveau spectre.

\section{a - Les multijonctions}

Dans un dispositif classique, les photons dont la conversion énergétique est la plus efficace sont ceux dont l'énergie est légèrement au-dessus du seuil d'absorption (gap). Pour ceux-ci, des rendements de l'ordre de $60 \%$ sont atteints expérimentalement, et sont d'ailleurs assez proches de ce que l'on peut attendre (fig. 2). L'utilisation de plusieurs cellules de gaps différents, chacune optimisée pour une partie différente du spectre solaire, permet donc en principe d'augmenter le rendement (voir encadré, p. 21). Pour un nombre donné de cellules et un spectre d'insolation fixé, il existe un choix optimal des 
gaps donnant le rendement le plus élevé [2] : par exemple, pour trois cellules sous concentration maximale, le rendement théorique maximum est de $63 \%$ (49\% sans concentration).

Ces dispositifs, parfois appelés " tandems", fonctionnent déjà, et ont démontré des rendements de $43 \%$ (sous concentration) [3]. Ils sont extrêmement sophistiqués dans leur réalisation et dans leur utilisation, mais c'est le premier type de dispositifs à potentiel de rendement élevé effectivement réalisé. C'est toujours aujourd'hui la seule manière démontrée d'obtenir des rendements de conversion de l'énergie solaire supérieurs à $30 \%$.

Les rendements les plus élevés ont été obtenus avec des structures basées sur des empilements de composés III-V en épitaxie. Des dispositifs tandem de grande taille comportant 2 et 3 jonctions ont aussi été réalisés avec des modules à base de couches minces de silicium amorphe, mais leurs rendements restent inférieurs à $15 \%$.

En fait, le gain incrémental de puissance acquis par l'ajout d'une cellule dans un tandem comprenant $\mathrm{N}$ jonctions, varie comme $1 / \mathrm{N}^{2}$. Si l'on prend en compte les imperfections du système, le gain espéré de l'ajout d'une cellule supplémentaire est voisin de zéro dès la 4e cellule.

\section{b - Les " transformateurs" optiques}

Une voie alternative consiste en l'interconversion de photons en amont du dispositif, de manière à obtenir un spectre plus étroit en énergie.

Considérons d'abord l'approche " addition de photons ». Les photons dont l'énergie est trop faible pour être utilisés directement par une cellule photovoltaïque classique, pourraient être en principe convertis par un dispositif d'optique non linéaire en un nombre plus faible de photons d'énergie plus grande. L'ensemble des photons de haute énergie est alors dirigé vers une cellule classique efficace dans ce domaine spectral.

Les principales recherches sur le sujet se font en utilisant des matériaux dans lesquels un rayonnement infrarouge est absorbé par plusieurs ions d'une terre rare, qui transferent ensuite de manière cohérente leur énergie à un autre lanthanide, capable d'émettre efficacement à la fréquence double [4].

Les quelques tentatives faites jusqu'ici ont prouvé la faisabilité du concept, mais avec un succès limité en termes de gain d'efficacité absolue : environ $3 \%$ des photons dans l'infrarouge dans la bande étroite d'absorption des terres rares utilisées, sous une illumination équivalente à 250 soleils, ont été effectivement convertis en photons de plus haute énergie.

Le principe de la "division de photons " consiste, à l'inverse, à absorber des photons de haute énergie dans un convertisseur luminescent pour émettre des photons de plus basse énergie, mais en plus grand nombre, vers une cellule solaire dont le seuil d'absorption est adapté. Le convertisseur fluorescent (e.g. $\mathrm{YF}_{3}: \mathrm{Pr}$ ou $\mathrm{LiGdF}_{4}: \mathrm{Eu}$ ) peut être placé en face avant, et avec un bon confinement optique ; l'essentiel de la lumière émise est absorbée par la cellule.

\section{II- Les matériaux à mécanismes d'absorption optimisés}

a - Les dispositifs à niveaux intermédiaires

Outre les transitions de la bande de valence à la bande de conduction qui ont lieu dans les semi-conducteurs sous l'effet du rayonnement, certains matériaux peuvent absorber des photons de plus basse énergie via des niveaux intermédiaires situés dans la bande interdite, qui jouent le rôle d'une "échelle à électrons » (fig. 3a) [5].

L'un des grands avantages de l'utilisation de ces matériaux est la réalisation d'un dispositif analogue à une multijonction pour ce qui est des rendements, mais avec la simplicité de l'élaboration d'une simple jonction. Une bonne absorption des niveaux intermédiaires suppose qu'ils soient à moitié occupés (c'est malheureusement aussi la condition pour la vitesse de recombinaison la plus élevée). Il faut donc chercher des systèmes dans lesquels ces niveaux ont un caractère "métallique", sans pour autant trop accroitre la vitesse de recombinaison non radiative. a

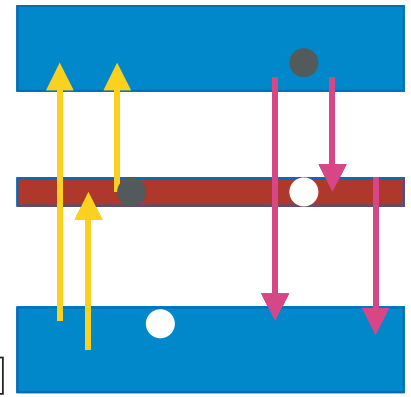

Bande Intermédiaire

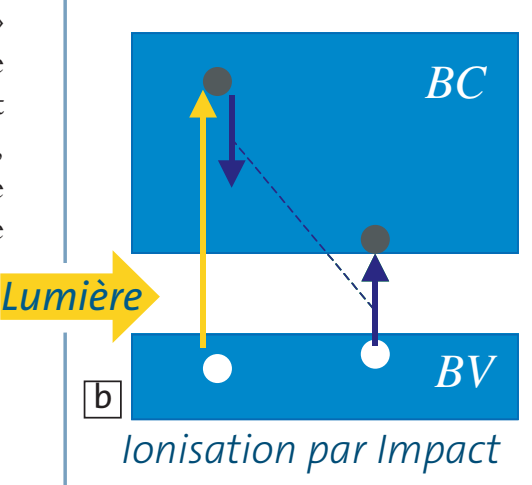

Figure 3 : Diagrammes de bande représentant les phénomènes de génération de porteurs optimisés par rapport au cas standard.

a) En haut, un matériau dans lequel une bande intermédiaire permet une meilleure absorption du spectre solaire. Les processus de génération sont en jaune et ceux de recombinaison sont en rose.

b) En bas, le phénomène d'ionisation par impact permet de transférer l'énergie excédentaire d'un porteur à un électron qui devient capable de passer de la bande de valence (BV) à la bande de conduction (BC).

: électrons

$O$ : trous

\section{Principe de la conversion multispectrale [triple jonction]}

a/ Un dispositif optique sépare la lumière solaire au moyen de filtres en trois faisceaux " bleu ", " vert " et " rouge ", qui sont convertis par trois cellules dont les bandes interdites ont été adaptées pour ces trois bandes spectrales. On peut simplifier le dispositif comme indiqué en b/ en remarquant que la cellule « bleue " n'absorbe pas les longueurs d'onde supérieures à son seuil d'absorption. Elle peut donc servir de filtre pour les cellules « vertes » et "rouges ". De la même façon, la cellule " verte " laisse passer le " rouge ". Il faut, bien entendu, que les substrats des deux premières cellules soient transparents. Enfin, en c/ on réalise un contact électrique entre les trois cellules. On peut réaliser cet empilement par dépôt des différentes cellules sur un même substrat. Pour éviter qu'il se crée une diode antagoniste à l'interface entre deux cellules (une diode $p$ - $n$ si on empile des diodes $n-p)$, on les relie souvent par une jonction tunnel. Dans le cas c/ les cellules sont connectées en série, alors que dans les cas a/ ou b/ on reste libre d'utiliser l'électricité produite de la façon la plus efficace.

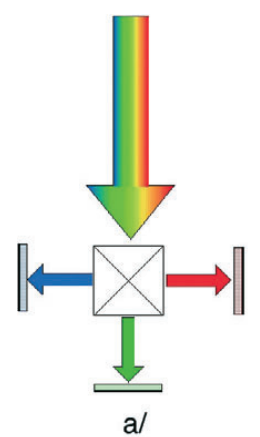

a/

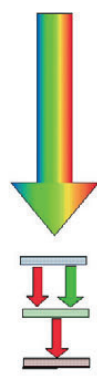

b/

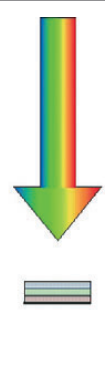

c/ 


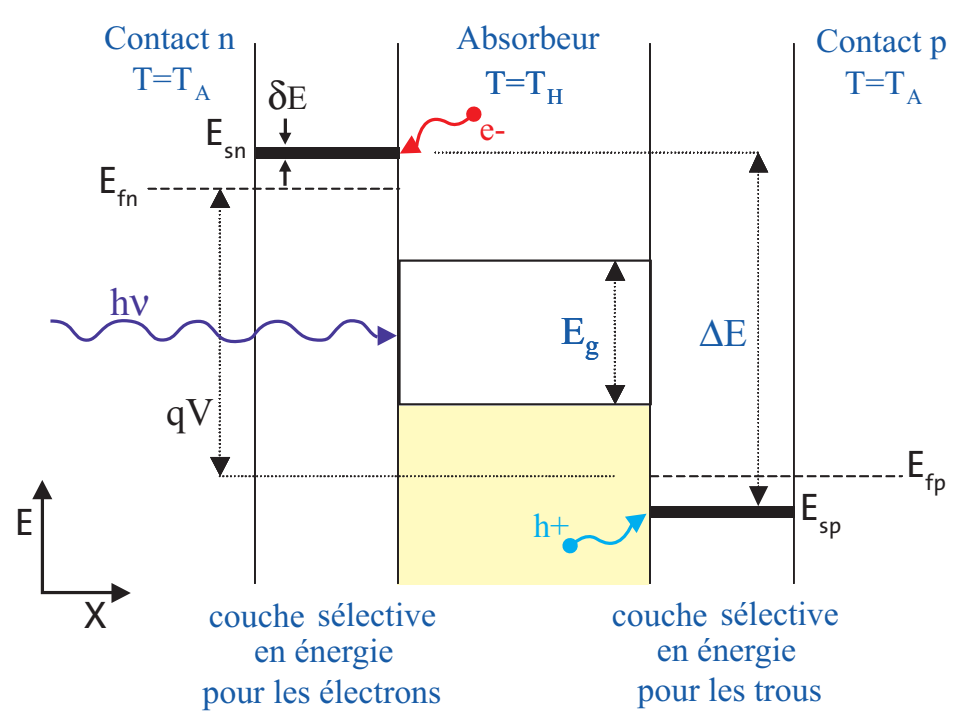

Figure 4 : Diagramme de bandes d'énergie d'une cellule à porteurs chauds. Les photons solaires sont absorbés dans la partie centrale où se forme un gaz d'électrons chauds (à une température $T_{H}$ supérieure à celle du réseau). Leur excédent d'énergie cinétique leur permet d'être récupérés à une énergie supérieure à celle du bord de bande de conduction (pour les électrons) ou de valence (pour les trous). La sélectivité en énergie $\delta E$ du contact est la condition pour que la distribution de porteurs chauds chauffe les contacts le moins possible.

\section{Références}

[1] W. Shockley et H.J. Queisser, J. Appl. Phys., 32 (1961) 510.

[2] M.A. Green, Third Generation Photovoltaics: Advanced Solar Electricity Generation, Springer, Berlin (2003).

[3] Nouveau record triple jonction de l'Université de Delaware à 42,8 \% : www.renewableenergyaccess.com $/ \mathrm{rea} / \mathrm{news} /$ story? $\mathrm{id}=49483 \& \mathrm{src}=\mathrm{rss}$ [4] P. Gibart et al., Proceedings of the $13^{\text {th }}$ Photovolt. Sol. Energy

Conference, Kluwer (1995), p. 85.

[5] A. Luqué et A. Marti, Phys.

Rev. Lett. 78 (1997) 5014.

[6] J.M. Raulot, C. Domain et J.F. Guillemoles, Phys. Rev. B 71 (2005) 35203.

[7] J.H. Werner, S. Kolodinski et H.J.Queisser, Phys. Rev. Lett. 72 (1994) 3851.

[8] R.T. Ross et A.J. Nozik, J. Appl. Phys. 53 (1982) 3813.

[9] Y. Rosenwaks et al., Phys. Rev. B 48 (1993) 14675.
Il existe de nombreuses manières d'obtenir des niveaux ou des bandes intermédiaires comme, par exemple, par l'introduction de défauts étendus ou d'impuretés, ou d'un super-réseau de plots quantiques.

D'autres systèmes à bandes intermédiaires, apparus plus récemment, paraissent intéressants. Il s'agit de composés semi-conducteurs ferromagnétiques, du type de GaAs:Mn, qui pourraient présenter des durées de vies des états intermédiaires élevées, à cause des règles de sélection sur le spin pouvant ralentir certains processus de recombinaison [6].

\section{b - Les matériaux " scintillateurs"}

L'absorption de photons dont l'énergie est plus de deux fois celle du gap permet d'envisager d'autres mécanismes que la création de phonons pour la thermalisation des paires électron-trous : l'excédent d'énergie peut servir à créer une seconde paire électron-trou, un phénomène nommé "ionisation par impact " (fig. 3b).

Les rendements limites de dispositifs à ionisation par impact peuvent être intéressants, à condition que le processus soit efficace au voisinage du seuil minimal $\left(\sim 2 \mathrm{E}_{\mathrm{g}}\right)$. Des travaux au Max Planck Institute de Stuttgart ont prédit que les alliages Si-Ge pourraient montrer un effet mesurable. Mais il s'est avéré inférieur à $1 \%$ du rendement de cellules de ce matériau. Des valeurs élevées d'ionisation par impact ne sont observées que pour des énergies de photons supérieures à $3 \mathrm{E}_{\mathrm{g}}$ [7].

\section{III - Les " machines thermiques" : cellules à porteurs chauds}

Les porteurs générés dans l'absorbeur ne se thermalisent pas instantanément avec le réseau à la température $T_{A}$, mais forment de manière transitoire un gaz d'électrons et de trous " chauds ": leur distribution correspond à une température $\mathrm{T}_{\mathrm{H}}>\mathrm{T}_{\mathrm{A}}$. Si ces porteurs peuvent être collectés rapidement via des niveaux étroits en énergie $E_{s n}$ et $E_{s p}$ (fig. 4), les flux de chaleur avec les contacts sont minimaux et la transformation de l'énergie cinétique du gaz chaud en énergie potentielle électrique est optimale [8].

Les calculs de la limite de rendement donnent des valeurs très proches de celles obtenues avec un dispositif multijonction contenant une infinité de cellules, chacune adaptée à une fraction du spectre, et ce pour un système de conception beaucoup plus simple : il s'agit donc, en quelque sorte, du dispositif ultime de conversion de l'énergie solaire.

Aucune cellule à porteurs chauds n'a encore été réalisée ; cependant, des mesures effectuées dans les semi-conducteurs usuels montrent que les porteurs chauds se thermalisent en quelques picosecondes. Dans les années 90 , on a découvert que le temps de thermalisation est fortement influencé à la fois par le niveau d'injection (i.e. l'intensité de l'excitation lumineuse) et par des effets de confinement [9]. Il apparait donc que les vitesses de thermalisation peuvent saturer dans des nanostructures à un niveau suffisant d'illumination. On prévoit alors des rendements de conversion qui pourraient dépasser $50 \%$ sous concentration. Ces rendements sont très sensibles à la largeur énergétique des contacts au-delà de quelques meV, car ces derniers introduisent une perte thermique par transfert de chaleur. Plus que la vitesse de thermalisation, la réalisation de contacts efficaces risque d'être le point délicat dans la fabrication de ces dispositifs.

\section{Conclusion}

La découverte de systèmes radicalement différents des diodes semi-conductrices pour la conversion à haute performance de l'énergie solaire est-elle une utopie ? Il existe en tout cas un grand nombre de possibilités pour y parvenir.

À court terme, les dispositifs à multijonction devraient faire des progrès significatifs et peut-être même avoir un impact notable dans la production d'électricité photovoltaïque, en utilisant une optique de concentration. À moyen terme, on envisage la réalisation de dispositifs optiques à addition d'énergie. Enfin, à plus long terme, les cellules à niveaux intermédiaires ou à porteurs chauds, qui posent les plus grandes difficultés scientifiques et technologiques, pourraient nous permettre d'approcher les rendements ultimes.

On peut espérer que ces possibilités verront le jour prochainement, grâce à la découverte de nouveaux matériaux, dont, mais pas seulement, ceux issus des nanotechnologies. Dans ce monde assoiffé d'énergie, il paraît nécessaire de tenter l'aventure.. 